\title{
Entscheidend ist, was hinten rauskommt
}

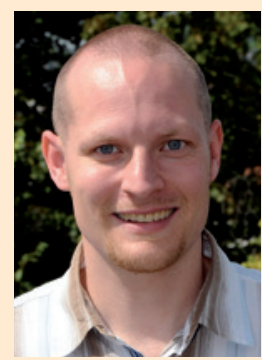

Christian Schäfer, Stuttgart
In diesen Tagen und in den nächsten Wochen rücken die Organspende und die Transplantationsmedizin wieder vermehrt in den Fokus der Öffentlichkeit. Das hat mehrere Gründe: Zum einen hat die Deutsche Stiftung Organtransplantation (DSO) an dem Tag, an dem ich diese Zeilen schreibe, bekannt gegeben, dass die Qualitätsberichte der Transplantationszentren für 2013 online stehen. Auf www.dso.de kann man die Ergebnisse abrufen, die durch Grafiken und die Möglichkeit, die Daten nach Transplantationszentren, DSO-Regionen, Bundesländern oder Organen bündeln zu lassen, leicht verständlich und übersichtlich sind. Dr. Axel Rahmel, Medizinischer DSO-Vorstand, meinte hierzu: „Die transparente und verständliche Darstellung der Qualität der Transplantationsergebnisse sind insbesondere für die Betroffenen und ihre Angehörigen wichtig, denn sie liefern unterstützende Informationen im Vorfeld einer Transplantation." Und nicht nur dort - je mehr Offenheit und Transparenz in diesem Thema herrschen, umso eher lässt sich die Vertrauenskrise, in der sich derzeit alles rund um die Organspende und Transplantationsmedizin befindet, bewältigen. Von daher ist die Art und Weise der Veröffentlichung der Qualitätsberichte grundsätzlich positiv zu bewerten.

Eine weitere Sache, die derzeit wieder mehr Medienrummel abbekommt, ist der Prozess um den sogenannten „Göttinger Transplantationsskandal“: Die Urteilsverkündung in diesem langen Verfahren ist für den 6. Mai geplant - wenn Sie diese Ausgabe der Dialyse aktuell in den Händen halten, wissen Sie also hierzu wahrscheinlich schon viel mehr als ich Ende April. Der Ausgang dieses Prozesses wird wegweisend für weitere Verfahren sein, die mit möglichen Manipulationen der Warteliste für Spenderorgane in anderen deutschen Transplantationszentren zu tun haben.

Welches Ergebnis am Ende auch vorliegt: Wichtig wäre es, dann die Konsequenzen weiter transparent aufzuarbeiten, um das Thema irgendwann in nicht allzu ferner Zukunft ad acta legen (sprich aus dem Fokus der Öffentlichkeit bringen) zu können. Denn wie ich schon in diversen Editorials der Vorjahre erläutert habe, sind Maßnahmen gegen Manipulationsmöglichkeiten bzgl. der Warteliste sowie der symbolträchtige Umbau der DSO längst initiiert und umgesetzt. Was jetzt zählt, ist der Rückgewinn des Vertrauens der Bevölkerung! Hierfür müssen die Verbesserungen noch mehr sowie transparent, gut und häufig kommuniziert werden. Denn letztendlich geht es darum, die Menschen von der Sinnhaftigkeit der Organspende zu überzeugen und somit mehr Spenderorgane zur Verfügung zu haben - denn der Mangel ist eklatant. Wie sagte dereinst Altbundeskanzler Dr. Helmut Kohl (CDU)? Richtig: „Entscheidend ist, was hinten rauskommt“ - in diesem Fall mehr Menschen, die nach dem für alle gleichen Prinzip der Wartelistenpriorisierung ausgesucht worden sind und durch ein neues Organ die Chance auf ein besseres und längeres Leben erhalten.

In diesem Zusammenhang ist der „Tag der Organspende“ am 6. Juni ein weiteres wichtiges Ereignis: U.a. über das bewährte Motto „Richtig. Wichtig. Lebenswichtig“ wollen die Veranstalter mit Vorurteilen aufräumen und zeigen, dass das Setzen eines Kreuzes im Organspendeausweis für andere Menschen unheimlich viel bedeuten kann. Die zentrale Großveranstaltung findet dieses Jahr auf dem Kröpcke in Hannover statt.

Auch in dieser Ausgabe der Dialyse aktuell können Sie etwas zum Thema Transplantation lesen: Auf Seite 184 präsentiert sich der Arbeitskreis Transplantationspflege e.V. (AKTX-Pflege) in einem neuen Gewand: Der Verein hat das Logo aufgefrischt, die Homepage neu aufgesetzt und einen komplett neu besetzten Vorstand. Innerhalb des aktuellen Schwerpunktthemas „Therapeutische Apherese“ beschäftigt sich außerdem einer der 3 Beiträge u. a. mit der Anwendung derselben bei der Nierentransplantation. Ich wünsche Ihnen eine angenehme Lektüre dieser Ausgabe der Dialyse aktuell! 